\title{
L'approche du décrochage scolaire selon les théories culturalistes de l'activité. Bénéfices et perspectives
}

An approach to school dropout according to culturalist activity theories. Profits

and prospects

Éric Flavier et Jacques Méard

\section{(2) OpenEdition}

Journals

Édition électronique

URL : http://journals.openedition.org/activites/2781

DOI : 10.4000/activites.2781

ISSN : 1765-2723

Éditeur

ARPACT - Association Recherches et Pratiques sur les ACTivités

Référence électronique

Éric Flavier et Jacques Méard, «L'approche du décrochage scolaire selon les théories culturalistes de l'activité. Bénéfices et perspectives », Activités [En ligne], 13-2 | 2016, mis en ligne le 15 octobre 2016, consulté le 19 avril 2019. URL : http://journals.openedition.org/activites/2781 ; DOI : 10.4000/ activites. 2781

Ce document a été généré automatiquement le 19 avril 2019

\section{cc) $(\mathrm{i})(9)$}

Activités est mis à disposition selon les termes de la licence Creative Commons Attribution - Pas d'Utilisation Commerciale - Pas de Modification 4.0 International. 


\section{L'approche du décrochage scolaire selon les théories culturalistes de l'activité. Bénéfices et perspectives}

An approach to school dropout according to culturalist activity theories. Profits and prospects

Éric Flavier et Jacques Méard

\section{NOTE DE L'ÉDITEUR}

Article soumis le 9 octobre 2015, accepté le 18 mai 2016

\section{Introduction}

1 La question de l'accrochage scolaire des élèves constitue l'une des principales préoccupations des politiques éducatives des pays industrialisés. Celles-ci ont fait du décrochage scolaire (DS) l'un des indicateurs clés de leur efficacité. Ainsi, le récent dossier d'actualité de l'Institut Français de l'Éducation (Thibert, 2013) fait état de l'intérêt largement partagé pour la cause de la lutte contre le DS, enjeu majeur des politiques publiques en matière d'éducation (Blaya, 2013). Les sphères institutionnelles et professionnelles en France relaient cet engouement par une littérature prolixe sur le sujet. Dans une récente recension, Bruno, Méard et Walter (2014) ont identifié 22 textes institutionnels, prescrivant 24 dispositifs de lutte contre le DS en France, dont neuf publiés après janvier 2011, ce qui révèle une volonté d'accélération de la prise en charge du DS.

Dans les travaux scientifiques, plusieurs typologies sont proposées dans le but de caractériser les populations plus ou moins à risque (Bautier, 2003 ; Bonnery, 2007 ; Hugon, 2010 ; Janosz, 2000), et ainsi cibler les actions de détection et de prévention du DS. Le 
phénomène y est décrit non comme un état, mais comme un processus complexe dépassant le strict cadre de la difficulté scolaire. Selon Millet et Thin (2005), la responsabilité d'une telle situation est imputable, de manière partagée, à l'élève, sa famille et son environnement social et scolaire. Ainsi ce processus de désadhésion progressive au travail scolaire (Blaya \& Hayden, 2003) semble résulter de l'influence simultanée de plusieurs facteurs scolaires (Baker, Derrer, Davis, Dinklage-Travis, Linder, \& Nicholson, 2001; Lee \& Burkham, 2003) et extrascolaires tels que les facteurs sociaux, économiques, familiaux (Cairns, Cairns, \& Neckerman ; 1989 ; Entwisle, 1990). Face à ce constat, l'école apporte deux formes de réponse. La première consiste, en fonction des élèves, en un effort d'adaptation des cursus et modalités d'accompagnement scolaire: prise en charge des troubles de l'apprentissage, identification de contenus d'enseignement ou de démarches d'enseignement dans le but de surmonter les difficultés des apprenants. Le second type de réponse au DS prend la forme d'un développement de partenariats éducatifs, notamment avec les parents ou les structures de prise en charge des élèves en dehors de l'école (Gilles \& Tièche Christinat, 2013). Mais, force est de constater que les adaptations préconisées par la littérature professionnelle et institutionnelle, ainsi que les dispositifs impliquant des partenaires, butent encore sur l'opacité du processus qui, en classe, conduit au décrochage (Bruno, et al., 2014).

Ces études convergent sur un certain nombre de principes relatifs à la prévention du DS : l'inscription de dispositifs sur le long terme, la détermination d'objectifs précis, l'évaluation des résultats des actions menées ou encore l'implication d'équipes éducatives cohérentes (Blaya, 2014). Mais, ces éléments n'épuisent pas la compréhension des modes d'émergence et de développement du processus de décrochage. Houflort et Sauvé (2010) soulignent par exemple la difficulté récurrente à comprendre finement ce qui amène un élève à se désengager du travail scolaire.

4 Afin de progresser dans cette compréhension, notons des travaux qui ne portent pas spécifiquement sur le DS mais plutôt sur les difficultés d'apprentissage. Ces travaux se revendiquant d'une épistémologie didactique ont montré que les élèves perçoivent les situations scolaires comme étanches, c'est-à-dire qu'ils ne peuvent trouver les ressources pour réussir la tâche demandée que dans la situation dans laquelle ils sont engagés, et particulièrement auprès du professeur (Margolinas \& Laparra, 2009). Les situations scolaires, initialement pensées pour rendre ce savoir accessible, induisent alors une certaine forme de fragmentation de ce dernier (Toullec-Théry \& Marlot, 2013). Cette stratégie d'enseignement, consistant à isoler les acquisitions attendues des élèves dans des tâches simplifiées et décontextualisées, se justifie par la volonté de les faire réussir et de maintenir une relation didactique avec eux (Marlot \& Toullec-Théry, 2011). Or, cette quête de la réussite scolaire à tout prix n'est pas sans conséquence sur le parcours des élèves. Pour Assude, Feuilladieu et Dunand (2015), la réduction du risque de DS implique d'accompagner les élèves dans le changement de leur rapport au savoir dans le cadre d'un enseignement rompant avec le cadre scolaire traditionnel. Les auteurs préconisent l'introduction d'innovations pédagogiques ainsi que le recours au numérique (Poyet, 2009 ; Schaumburg, Prasse, Tschackert, \& Blömeke, 2007) ou encore aux jeux sérieux (Sanchez, 2009).

5 Néanmoins, ces études privilégient une démarche centrée sur l'identification des savoirs en jeu dans la relation d'apprentissage. Or, Brown et Rodriguez (2009) pointent la difficulté à distinguer clairement les facteurs scolaires des facteurs extrascolaires tant ils apparaissent imbriqués dans l'activité in situ des acteurs. Dès lors, pour certains auteurs, 
la question de la nature, de la forme, de la signification de l'activité des élèves et des professionnels de l'éducation pour comprendre le phénomène du DS devient prioritaire (Flavier \& Moussay, 2014).

\section{L'objectif de l'article}

Dans ce contexte, notre but est ici d'appréhender la problématique de la lutte contre le DS au plus près des acteurs et de leurs pratiques selon une approche "orientée activité " (Durand, de Saint-Georges, \& Meuwly-Bonte, 2006), plus précisément celle qui se revendique de la Cultural Historical Activity Theory (CHAT) (Engeström, 2001; Leontiev, 1984 ;Vygotski, 1934/1997). Dans la mouvance des conceptions récentes en formation professionnelle des adultes (Yvon \& Durand, 2014), notamment des enseignants, nous soutenons ici qu'il est utile, en complément des autres voies de compréhension, de développer ces travaux relatifs à la lutte contre le DS selon cette approche. À partir de ces postulats théoriques, nous déclinerons trois hypothèses spécifiques de ce courant qui semblent à même de renouveler les angles d'analyse du processus de DS et offrir des perspectives d'étude concernant sa prévention : l'hypothèse d'un développement par le sens et l'efficience, l'hypothèse d'un développement par des motifs concurrents, l'hypothèse d'un développement dans un « système d'activité ».

\section{Méthode}

7 Cette discussion prendra appui sur les résultats d'un certain nombre de travaux empiriques qui, bien qu'ils aient essentiellement ici une fonction de clarification et d'illustration, méritent un éclaircissement méthodologique préalable. Plus précisément, les extraits et résultats présentés ici sont issus de trois études récentes : a) Étude 1 : étude des interactions enseignant-élèves (Méard, 2014); b) Étude 2: Étude du dispositif «Enterprise virtuelle» (Flavier, 2014); c) Étude 3 : Étude du dispositif "Point élève » (Flavier, Moussay \& Méard, 2015). Le recueil de données s'est déroulé au cours d'une année scolaire au sein de quatre collèges français. En s'inspirant des principes méthodologiques de la clinique de l'activité (Clot, 1999), trois types de données ont été recueillis. Dans un premier temps, des données ethnographiques (observations directes, entretiens informels réalisés auprès des élèves ou des professionnels) ont été collectées afin de découvrir le contexte et établir un contrat de recherche avec les acteurs. Des données d'observation et d'enregistrement audio-visuel des situations de classe (cours disciplinaires ou séquences avec les élèves autour de projets particuliers) ou des situations de travail (réunions d'équipe pédagogique ou avec des partenaires éducatifs) ont été l'objet d'un deuxième temps de recueil (Tableau 1). Des données de verbalisation $a$ posteriori ont été recueillies et enregistrées; elles consistaient en des entretiens d'autoconfrontation simple avec les enseignants, les CPE (conseillers principaux d'éducation) et les autres acteurs de la communauté éducative (assistante sociale scolaire, conseillère d'orientation psychologue). Lorsque les traces audiovisuelles d'activité n'avaient pu être collectées, nous avons eu recours à des entretiens de type instructions au sosie (Tableau 1). Ces données de verbalisation visaient à accéder à l'activité des acteurs et à « forcer leur développement ». 
Tableau 1 : Bilan quantitatif des données recueillies.

Table 1 : Quantitative assessment of collected data

\begin{tabular}{|c|c|c|}
\hline & Données d'observation & Données de verbalisation a posteriori \\
\hline $\begin{array}{l}\text { Étude } 1 \\
\text { (Méard, 2014) } \\
\text { Étude des interactions } \\
\text { enseignant-élèves }\end{array}$ & - 82 leçons en classe & $\begin{array}{l}\text { - } 14 \text { autoconfrontations simples } \\
\text { avec les enseignants participant à } \\
\text { l'étude } \\
\text { - Entretiens compréhensifs avec } \\
\text { élèves et/ou enseignants } \\
\text { consécutifs aux observations }\end{array}$ \\
\hline $\begin{array}{l}\text { Étude } 2 \\
\text { (Flavier 2014) } \\
\text { Étude du dispositif } \\
\text { «Enterprise virtuelle» }\end{array}$ & $\begin{array}{l}\text { - } 2 \text { réunions de concertation de } \\
1 \text { 'équipe pédagogique } \\
\text { - } 2 \text { conseils de classe } \\
\text { - } 16 \text { séquences en classe (leçon ou } \\
\text { séquence projet) }\end{array}$ & $\begin{array}{l}2 \text { entretiens « instruction au } \\
\text { sosie » avec chacun des } 2 \text { co- } \\
\text { porteurs du projet } \\
2 \text { entretiens « instruction au } \\
\text { sosie » avec } 2 \text { enseignants }\end{array}$ \\
\hline $\begin{array}{l}\text { Étude } 3 \\
\text { (Flavier et coll, 2015) } \\
\text { Étude du dispositif } \\
\text { «Point élève" }\end{array}$ & $\begin{array}{l}\text { - } 19 \text { réunions du groupe « point } \\
\text { élève " }\end{array}$ & $\begin{array}{l}1 \text { entretien compréhensif }+1 \\
\text { instruction au sosie avec chacun } \\
\text { des } 4 \text { acteurs suivant : les } 2 \\
\text { conseillers principaux } \\
\text { d'éducation, la conseillère } \\
\text { d'orientation psychologue, } \\
\text { l'assistante sociale scolaire }\end{array}$ \\
\hline
\end{tabular}

8 Le traitement des données portait dans un premier temps sur les données d'entretiens, selon un protocole en trois étapes (Flavier, Moussay \& Méard, 2015) : a) un découpage en unités d'analyse à partir de l'identification des buts d'action; b) un codage visant à dégager les buts d'actions, les opérations et les motifs d'agir en référence au modèle de Leontiev (1984); c) un codage visant à dégager les pôles Objet-Outils-RèglesCommunauté-Division du travail, en référence au modèle d'Engeström (2001) (voir le Tableau 2). Ensuite, pour chaque acteur, des triangulations ont été opérées avec les autres données (observations...) pour reconstituer un processus éventuel de développement à partir d'indicateurs. Enfin, des comparaisons entre acteurs ont permis de pointer des tensions dans les systèmes d'activités. 
Tableau 2 : Illustration du traitement des données d'entretien.

Table 2 : Illustration of interview data processing

\begin{tabular}{|c|c|c|}
\hline Extrait d'entretien & Codage 1 & Codage 2 \\
\hline $\begin{array}{l}117 \mathrm{Ch} \text { : Quand tu présentes le cas au « point- } \\
\text { élève " tu cibles les informations } \\
118 \mathrm{SM} \text { : Oui } \\
119 \mathrm{Ch}: \text { A partir de quoi } \\
120 \mathrm{SM} \text { : Ben par exemple au niveau culture } \\
\text { éducation nationale heu on va éviter de parler } \\
\text { de certains trucs donc je vais le dire qu'à } \\
\text { l'infirmière ou au chef d'établissement } \\
121 \mathrm{Ch}: \text { Il y a des informations qui n'ont pas } \\
\text { lieu d'être au point-élèves } \\
122 \text { SM : Qui n'ont pas lieu d'être ou qui } \\
\text { peuvent être considérées comme ne relevant } \\
\text { pas du point-élèves tout dépend des personnes } \\
\text { des personnalités qui sont là maintenant heu, il } \\
\text { y a aussi autre chose, au collège je suis CPE je } \\
\text { ne suis plus éducatrice spécialisée donc je vais } \\
\text { me contraindre à être seulement dans la peau } \\
\text { du CPE pour éviter de créer des tensions de la } \\
\text { rivalité avec une infirmière avec une } \\
\text { assistante sociale avec les éducateurs } \\
123 \text { Ch : Les informations que tu livres au } \\
\text { point-élèves sont les informations du CPE } \\
124 \text { SM : i'essaye d'être le plus carré le plus } \\
\text { pragmatique possible je vais dire je vais être } \\
\text { très méthodique ça va être le nom la date de } \\
\text { naissance les antécédents souvent heu les } \\
\text { antécédents scolaires la problématique que le } \\
\text { CPE relève ce qui a été fait au niveau de la } \\
\text { remédiation }\end{array}$ & $\begin{array}{l}\text { Motif : ne pas créer de } \\
\text { tensions avec l'infirmière, } \\
\text { l'assistance sociale, les } \\
\text { éducateurs } \\
\text { Opération : être carrée, } \\
\text { pragmatique, méthodique } \\
\text { dans la présentation du cas- } \\
\text { élève } \\
\text { Opération : être dans la } \\
\text { problématique du CPE dans } \\
\text { la présentation du cas-élève }\end{array}$ & $\begin{array}{l}\text { Communauté: au niveau } \\
\text { culture éducation nationale } \\
\text { Division du travail : je vais le } \\
\text { dire qu'à l'infirmière ou au } \\
\text { chef d'établissement } \\
\text { Division du travail - Règles: } \\
\text { Qui n'ont pas lieu d'être } \\
\text { Communauté : je suis CPE je } \\
\text { ne suis plus éducatrice } \\
\text { spécialisée }\end{array}$ \\
\hline
\end{tabular}

\section{Résultats}

\subsection{L'hypothèse d'un développement par le sens et l'efficience}

Dans les analyses fondées sur la CHAT, notamment l'approche développementale de la clinique de l'activité (Clot, 1999; 2008), c'est la dimension psychologique du développement au travail qui est considérée au premier plan. Pour le sujet qui nous intéresse, il s'agit donc d'appréhender les traces et les circonstances du développement de l'activité des élèves en situation de décrochage. Mais, dans la mesure où la CHAT postule une dimension culturelle (l'activité est médiée par des outils culturels) et sociale (les apprentissages et le développement des acteurs se déploient au cours d'interactions), le processus du DS de l'élève y est également compris au travers de l'activité des autres élèves et des enseignants.

10 De plus, pour Vygotski (1934/1997), l'action est toujours dirigée vers un but conscient, c'est-à-dire comme une « représentation cognitive du résultat à atteindre » (Clot, 1999, p. 169). À cette fin, l'acteur met en œuvre concrètement les moyens de son activité, moyens que Leontiev (1984) nomme "opérations », autrement dit les gestes techniques que l'acteur réalise effectivement pour agir et atteindre le but qu'il s'est fixé. Ces opérations sont « choisies » par lui parmi les possibles offerts, réalisables ou non, par les circonstances de l'environnement dans lequel il agit. Mais cette action dirigée vers un but est également sous-tendue par des «motifs", désignant «ce qui compte vraiment pour l'acteur », au sens profond et personnel qu'il donne à ses actions et qui le pousse à agir. L'idée centrale à ce niveau est que, « vidée de son sens, l'activité du sujet se trouve dévitalisée et amputée de son 
pouvoir d'agir" (Clot, 2008, p.13). Leontiev propose donc une structure de l'activité humaine articulée autour de trois composantes (le but de l'action, le motif et l'opération) ou, pour être plus précis, l'articulation entre ces trois composantes, c'est-à-dire les rapports entre le but et les opérations (l'efficience de l'activité) et entre le but et le motif (le sens de l'activité).

11 Ce postulat théorique fait envisager dès lors le DS des élèves comme un processus inhérent à une amputation du sens de l'activité et/ou de l'efficience dans les tâches prescrites par l'enseignant.

12 À ce propos, Méard (2014, p. 44) rapporte cet échange avec un collégien à risque de décrochage commentant son activité face à un ordinateur en classe sur un logiciel de géométrie lors d'une séquence relative au théorème de Pythagore :

Chercheur. : tu t'es arrêté de travailler tout à l'heure, tu te souviens? Tu t'es mis

comme ça sur ta chaise [mime un recul, les bras derrière la tête].

Élève : non, j'étais fatigué (motif). Les maths, y a pas que ça dans la vie [rire].

Chercheur: [rire], mais à ce moment tu te souviens? Tu t'es mis en arrière parce

que tu avais l'air énervé

Élève : ah oui, j'arrivais pas à... à faire tourner le triangle (opération)

Chercheur : à le faire pivoter sur l'écran?

Élève : ouais, j'ai cherché partout, j'ai demandé à Manu et puis ça m'a saoulé. J'ai préféré discuter avec $\mathrm{T}$.

13 Rendant compte de l'expérience vécue par cet élève, cet extrait illustre le processus de décrochage d'un élève en cours de mathématiques. D'abord mu par l'envie de réussir, l'élève persévère un moment puis finit par abandonner : «j'arrivais pas (...) j'ai cherché (...) j'ai demandé (...) ça ma saoulé». Dans le cas présent, le décrochage de l'élève est lié à l'inefficience des opérations qu'il met en œuvre - «j'arrivais pas à faire tourner le triangle »puis à un manque de persévérance dont on trouve les raisons dans l'examen des motifs d'agir de l'élève - «les maths, y a pas que ça dans la vie »- ne soutenant pas les efforts nécessaires à la réussite du travail demandé par l'enseignant. En d'autres termes, réussir la tâche prescrite n'a d'autres finalités, pour l'élève, que celle de la réussite scolaire en cours de mathématiques, ce qui n'est pas suffisant pour maintenir son engagement dans cette situation. Cette hypothèse énoncée avec les concepts de la CHAT décrit alors un cercle vicieux de décrochage alimenté par l'antinomie entre les motifs d'agir de l'élève et ceux attendus de lui par l'enseignant.

14 Dans les résultats des études fondées sur l'analyse d'activité, le processus peut parfois se transformer en un cercle vertueux indexé à des formes de travail qui soutiennent l'élève tantôt dans la construction du sens de son activité par l'indexation de celle-ci à de nouveaux motifs d'agir, tantôt dans l'appropriation de techniques et d'instruments jusqu'à présent non maitrisés (Flavier \& Moussay, 2014). Un tel processus s'apparente à ce que Clot (1999), à la suite de Leontiev (1984), identifie comme le processus de développement biphasé de l'activité "assurant le dynamisme des rapports entre motifs et moyens du sujet» (p.173). Les résultats d'une étude portant sur le dispositif nommé " entreprise virtuelle » proposé à une classe de $4^{\text {ème }}$ permettent d'illustrer ce type de dynamique de développement: ce dispositif avait pour objectif de fédérer un groupe classe autour d'un projet commun, celui de la création, virtuellement, d'une entreprise et de son implantation dans un bassin local. Les élèves de cette classe en risque de décrochage étaient invités à s'engager avec l'enseignant dans la réalisation de ce projet. À cette fin, les différents enseignements disciplinaires soutenaient le projet: l'élève apprenait à distinguer et maîtriser différents registres de langage en cours de français en 
travaillant sur les slogans publicitaires; il étudiait en art plastique le graphisme par la réalisation du logo, dessinait les plans et construisait une maquette en mathématiques tout en travaillant les volumes et la proportionnalité. En proposant aux élèves de nouvelles tâches, liées à la réalisation du projet commun, les enseignants leur suggéraient de nouveaux motifs d'agir susceptibles de recueillir leur adhésion, d'autant plus que ces nouveaux motifs étaient partagés entre enseignants et élèves. Après traitement des données, l'étude a mis en lumière que l'engagement durable d'élèves à risque de décrochage au cours de ce dispositif était en lien principalement avec la poursuite de ces nouveaux motifs - la conception des plans et de la maquette - qui impliquaient l'acquisition de nouveaux savoirs - ici relatifs aux volumes et à la proportionnalité. Tout autant en cours de mathématiques que lors des séquences "entreprise virtuelle», la maitrise des techniques de calcul et de proportionnalité était nécessaire à la réussite de l'élève dans les tâches demandées, entrainnant le développement de nouvelles " opérations », donc un gain d'efficience.

Ainsi, un déficit de sens peut être à l'origine du processus de DS parce qu'il déclenche, entretient ou accentue un désengagement de l'élève qui finit par ne plus agir (donc finalement à ne plus développer les opérations nécessaires à ses apprentissages et à son développement). Ce faisceau d'hypothèses en analyse d'activité rejoint, pour partie, celles du réseau RESEIDA qui conjugue des éclairages sociologiques et didactiques pour comprendre les modalités de construction des inégalités scolaires. Ainsi, selon Kahn (2010), les échecs répétés tendent à accentuer la difficulté de l'élève à appréhender la culture scolaire et participent ainsi de l'émergence de "malentendus socio-cognitifs » (Bautier \& Rochex, 1997 ; Broccolicchi, 2000). Selon ces auteurs, ces malentendus répétés sont à l'origine du processus de DS car ils accentuent les écarts entre les attentes réciproques de l'école d'une part et des élèves et des familles d'autre part; le social et les interactions sont alors compris comme des facteurs venant complexifier le triptyque (didactique) enseignant-savoir-élève dans la mesure où la relation pédagogique ne peut être appréhendée sans tenir compte de l'environnement dans lequel elle prend forme. Le travail de Margolinas et Laparra (2011) montre par exemple que certaines interactions décalées des élèves (par exemple lorsqu'ils posent des questions peu en rapport avec le cours dispensé) sont parfois judicieusement exploitées par les enseignants afin de maintenir les élèves en activité. Ce faisant, ce type de situation contribue pourtant à entretenir l'ambiguïté inhérente à ces malentendus sociocognitifs.

Sur ce point, si les résultats intéressants du réseau RESEIDA confirment certains aspects des études sur le DS en analyse d'activité, il existe néanmoins deux spécificités à cette dernière : d'une part, dans l'activité du sujet, la dimension cognitive ne se distingue pas des sphères volitive et émotionnelle (Vygotski, 1934/1997). Dès lors, le concept de malentendu «sociocognitif» devient restrictif car il relègue la dimension d'intentionnalité et d'émotion au second plan dans le processus de décrochage de l'élève. D'autre part, en analyse d'activité, l'interaction sociale est au centre du processus d'apprentissage et de développement des acteurs (et donc, en l'occurrence, du décrochage et de la persistance scolaire). Ce postulat décrit un processus dans lequel ce sont les divergences, les contradictions, les différences de motifs entre acteurs qui sont rapatriées dans l'activité du sujet sous forme de conflits intrapsychiques et qui sont à la source de ses apprentissages et de son développement. 


\subsection{L'hypothèse d'un développement par des motifs concurrents}

17 Cette spécificité de l'approche du DS par la CHAT conduit à présenter une deuxième hypothèse. En effet, le développement biphasé de l'élève (par le sens et l'efficience) ne peut être appréhendé qu'en interdépendance avec l'environnement social dans lequel elle se déploie. En d'autres termes, sur la base des interactions entre élèves et entre l'élève et l'enseignant, les actions de ce dernier en classe impactent, de manière indirecte, les formes d'engagement de l'élève par la recherche de motifs partagés. Cette préoccupation est récurrente dans les données des recherches en clinique de l'activité, par exemple chez cet enseignant d'anglais en collège :

Enseignant: faut toujours se demander ce qui va les accrocher [motif]. C'est le problème permanent... et tu es toujours sur le fil du rasoir, surtout avec certains gosses. En une seconde, ils ne sont plus là, ils lâchent le boulot, ils sont sur une autre planète. Tu risques de perdre ta classe en une seconde... Alors moi, je pense toujours à ça [motif].

Mais la suggestion de motifs partagés par l'enseignant ne produit pas toujours les effets escomptés. En témoignent les propos recueillis auprès de cet élève de $4^{\mathrm{ème}}$, assistant, avec bien peu d'engouement, à un cours de technologie sur le rapport entre l'économie d'énergie et l'isolation d'une maison.

Chercheur $(\mathrm{CH})$ : ça ne t'a pas tellement intéressé cette fois, on dirait.

Élève: (silence) ça ne m'intéresse pas. Je ne comprends pas à quoi ça sert [non accrochage aux motifs suggérés par l'enseignant].

Chercheur : l'isolation des toits, combien ça coûte, le gaspillage d'énergie... non ?

Élève : (silence) moi j'habite en appartement, j'ai pas de toit.

19 Ce court extrait illustre les tensions qui peuvent apparaitre dans la classe du fait de désaccords ou d'incompréhensions autour de l'objet de la leçon. Dans le cas présent, l'élève ne se reconnait pas dans l'effort de contextualisation proposé par l'enseignant pour rendre plus attractifs les apprentissages scolaires. Le lien qui unit les différents acteurs vers un motif partagé se délite par ce qui est considéré comme un manquement de l'autre dans la division du travail (pour reprendre la situation archétypale de Leontiev : le rabatteur de gibier ne rabattant plus, le chasseur arrête de chasser).

Au-delà de cet accrochage manqué aux motifs suggérés par l'enseignant, le processus de DS trouve aussi son origine dans les interactions dissimulées entre élèves. En d'autres termes, le collectif d'élèves et ses règles implicites pèsent sur la construction des motifs d'agir de chacun, conduisant, dans certaines circonstances, à un risque de décrochage. Par exemple, lors d'une leçon d'EPS, l'analyse de l'activité de l'élève $M$ révèle comme motif d'agir principal « (agir pour) ne pas échouer » :

Chercheur : Tu as sauté combien de fois en hauteur aujourd'hui?

Élève $\mathrm{M}:$ [silence]... Cinq ou six fois.

Chercheur: Tu es sûr?

Élève $\mathrm{M}:$ [silence]

Chercheur :J'ai eu l'impression que tu as sauté moins que ça, non?

Élève $\mathrm{M}$ : [silence] Je ne suis pas douée en saut en hauteur.

Chercheur: Moi non plus, je n'étais pas bon en hauteur. Mais bon, on est là pour apprendre.

Élève $\mathrm{M}:$ [silence]

[...]

Chercheur Ch: Qu'est- ce que tu en penses? Tout le monde peut progresser, non?

Élève $\mathrm{M}$ : Oui, si les garçons se moquaient pas, là j'voudrais bien essayer, mais là, je 
vois qu'ils rigolent, ces boloss.

Chercheur : Ils rigolent?

Élève $M$ : Oui, dès que M. R. n'entend pas, ils te disent « retourne en primaire pour sauter à l'élastique » et des trucs... et ils soufflent quand on baisse la barre parce que « Monsieur » il saute haut, il s'croit très fort.

Dans cette situation, la crainte d'échouer ou les moqueries à son encontre renforcent la validité de ce motif d'agir «(agir pour) ne pas échouer » et conduit progressivement l'élève $M$ à un désengagement de la leçon.

Ces interactions plus ou moins dissimulées au sein du collectif d'élèves opèrent finalement à la manière de prescriptions venant concurrencer celles de l'enseignant (les consignes relatives au travail scolaire). C'est également la situation vécue par cette autre collégienne en cours de technologie (Méard, 2014, p. 47).

Chercheur : je me demandais si tu étais intéressée par ce travail.

Élève : si, si, mais j'ai du mal pour apprendre mes leçons.

Chercheur : je me demandais ça aussi parce que tu ne lèves jamais la main quand $M$.

E pose des questions

Élève : [silence]

(...)

Chercheur : Tu ne sais pas répondre? Ou tu ne veux pas me répondre?

Élève : Si si des fois, mais j'ai peur...

Chercheur : de quoi?

Elève : de me tromper, de passer pour une idiote

Chercheur : et si tu réponds juste?

Élève : pareil

Chercheur : comment ça «pareil»?

Élève : ben, passer pour une idiote

Chercheur : ah, tu as peur que les autres se moquent de toi ?

Élève : oui.

Cet extrait illustre un aspect de l'activité de cette élève, prise en tenaille entre les prescriptions énoncées par l'enseignant, relatives au suivi et à l'engagement dans la leçon et celles émanant du collectif d'élèves et impliquant de ne pas s'engager trop ostensiblement dans la leçon. Le " coût» psychologique représenté par une éventuelle infraction aux règles du collectif d'élèves est tel pour cette élève qu'elle préfère enfreindre les prescriptions de l'enseignant. Pour le dire autrement, les motifs d'agir suggérés implicitement par le collectif d'élève, dans le cadre d'interactions dissimulées dans le temps de la leçon, s'imposent à ceux suggérés explicitement par l'enseignant.

Que les interactions avec les pairs soient des vecteurs de décrochage ou de persévérance, la question de l'engagement déborde largement les questions de rapport au savoir ou de rapport à l'enseignant, lorsqu'on analyse l'activité des élèves à risque de décrochage.

En résumé, trois phénomènes inhérents aux interactions participent du risque de DS : la non-reconnaissance par l'élève de la légitimité des motifs d'agir suggérés par l'enseignant, la prédominance de motifs d'agir antinomiques avec l'acte d'apprentissage (pour ne pas échouer) et la prédominance des motifs d'agir suggérés par le collectif d'élèves. Leur mise en exergue renforce l'idée que l'on ne peut se limiter à une analyse de la tâche ou de ce que l'élève réalise (au sens de ce qu'il montre à voir) pour comprendre le processus de DS. L'approche clinique de l'activité ouvre la voie d'une analyse complémentaire du DS en éclairant les soubassements collectifs et communautaires de ces interactions pour les acteurs respectifs. 


\subsection{L'hypothèse d'un développement de l'acteur dans un « système d'activité "}

Malgré les efforts d'anticipation des enseignants, le moment de la classe est celui des imprévus. Les contraintes qui s'exercent sur le métier d'enseignant (conditions de travail, empilement des prescriptions, complexité et simultanéité des problèmes à résoudre, délitement des collectifs de travail, diversité des élèves, etc.) impactent à leur tour le développement professionnel de celui-ci. Spécifiquement, les élèves à risque de DS posent des défis quotidiens majeurs aux enseignants en accentuant l'imprévisibilité, ce qui contribue à faire émerger et à entretenir une dynamique de décrochage professionnel (Karsenti \& Collin, 2009; Mukamurera \& Bouthiette, 2008). On observe ce phénomène d'abandon notamment chez les enseignants débutants qui nourrissent peu à peu le sentiment de ne pas réussir à faire leur métier dans les règles de l'art (Giust-Desprairies, 1996 ; Roger, 2007).

Donc, d'une part le travail de l'enseignant impacte le processus du DS de l'élève, d'autre part, ce décrochage de l'apprenant confronte l'enseignant à des contraintes fortes, caractérisées par l'émergence de situations d'empêchement ou de renoncement, mettant à mal la réalisation de son métier. Aussi, l'analyse clinique de l'activité des élèves décrocheurs et de leurs enseignants tend à accréditer l'hypothèse de deux processus consubstantiels: le DS de l'élève et le décrochage professionnel de l'enseignant. Le caractère prétendument interpénétré de ces deux processus, celui de l'adulte et celui de l'élève, résiderait dans les tensions repérables entre les motifs de l'un (l'enseignant ou l'élève) et les opérations de l'autre et réciproquement. Par exemple, il en est ainsi lorsque l'enseignant introduit une nouvelle règle qui vient en contradiction avec une autre règle précédemment établie, comme le montrent Moussay et Flavier (2014). Dans l'étude présentée par ces auteurs, l'enseignante refuse de donner la parole à un élève au motif qu'il n'avait pas levé la main alors que sa remarque était pertinente. Elle est alors en proie à des dilemmes professionnels qui alimentent un décrochage conjoint des acteurs. Cette consubstantialité des deux décrochages s'observe également dans les tensions entre les instruments et les objets de l'activité de l'élève initialement suggérés par l'enseignant d'une part et ceux que l'élève s'assigne réellement d'autre part. Un collégien en situation d'échec scolaire peut par exemple, à l'insu des enseignants, détourner la consigne relative à la recherche et au choix d'une orientation professionnelle en la remplaçant par une « découverte " professionnelle, une navigation exploratoire dont il perçoit l'opportunité ludique pour s'extraire de l'ennui produit par le travail demandé (ibid.). Ce faisant, il ne se développe pas, mais, dans ce cas, l'étude montre qu'il conduit aussi l'enseignant concerné à ressentir une impuissance à lutter contre le DS et à juger que, dans le système d'activité, cet élève «ne fait pas sa part » dans la division du travail, que « décidément, il ne joue pas le jeu » (dans la situation archétypale de Leontiev, le chasseur ne chassant plus, le rabatteur arrête de rabattre).

28 Dans les dernières avancées de la CHAT (Engeström, 2004), le développement du sujet s'inscrit dans un «système d'activité » qui englobe et met en relation plusieurs acteurs dans l'institution. Reprenant Vygotski, cet auteur insiste sur le rôle fondamental des «tensions » qui permettent aux acteurs de se donner de nouveaux buts, de nouveaux motifs d'agir. 
idérer le DS, non plus dans un contexte inter-individuel mais aussi dans un système d'activités collectives, apporte des éléments supplémentaires à la compréhension de la consubstantialité du DS de l'élève et du décrochage professionnel de l'enseignant. En effet, les études menées dans ce cadre conceptuel font voir la classe non plus comme un environnement hiérarchique où l'enseignant est seul responsable, mais plutôt comme une organisation émergente et autonome. Ces options théoriques amènent à rompre avec l'image d'un enseignant-décideur unique pour adopter le concept de knotworking (ibid.). Ce concept de «travail en nœuds » décrit une forme d'organisation multipolaire avec un centre de décision qui se déplace au gré des interactions.

Par exemple, dans le cadre du dispositif entreprise virtuelle évoqué plus haut, les enseignants et les élèves se retrouvent engagés conjointement dans un projet commun dont ni les premiers, ni les seconds ne maitrisent pleinement la destinée. Cela conduit les enseignants à certaines hésitations qu'ils partagent avec les élèves, à réfléchir avec eux pour construire véritablement avec eux les solutions aux problèmes rencontrés. Évoquant l'un de ces moments, un enseignant participant à ce dispositif confiait au cours d'un entretien que :

«[les élèves] voient bien que l'on [l'enseignant] ne sait pas tout, que l'on cherche parfois avec eux à construire ce dont on a besoin pour l'entreprise [virtuelle]. Ils ont des idées, ils proposent, ils décident $"$.

En faisant des élèves de véritables acteurs, l'enseignant modifie les modalités usuelles de division du travail dans la classe et rend alors perceptible à l'élève le processus de coconstruction du sens. Cette relative instabilité perdure sans générer de profonds déséquilibres grâce à un second phénomène décrit par Engeström : le boundary crossing (franchissement de frontières) qui définit les écarts que chacun s'autorise à faire quant aux prérogatives qui sont les siennes, avec le consentement tacite des autres. Par exemple, lorsque certains élèves imposent à leurs camarades de réaliser avec sérieux les tâches qui leur incombent dans le but de permettre la poursuite du projet commun dans de bonnes conditions.

31 À une autre échelle, le knotworking (travail en nœud) et le boundary crossing émergent dans les dispositifs collectifs de lutte contre le DS engageant plusieurs acteurs (enseignants, éducateurs, partenaires éducatifs) (Gilles \& Tièche-Christinat, 2013). C'est notamment le cas des dispositifs de veille et d'accompagnement des d'élèves repérés comme «à risque de DS ». Un tel dispositif a été étudié par Flavier, Moussay et Méard (2015) en collège. Il rassemble les différents acteurs de la communauté éducative élargie de l'établissement, dont certains comme l'assistante sociale, l'infirmière scolaire ou la conseillère d'orientation psychologue, sont soumis au secret professionnel. Ces derniers rencontrent alors des dilemmes caractérisés par la concurrence de motifs suggérés d'une part par le collectif des enseignants, d'autre part par le collectif professionnel de référence. Par exemple, l'assistante sociale est tiraillée entre la nécessité de délivrer des informations sur la situation familiale d'un élève pour faire avancer le débat collectif et l'interdiction de trahir un secret professionnel.

32 À titre d'illustration, l'assistante sociale (AS) énonçait au cours d'un entretien (ibid. p. 186) :

AS: Ca non je le dirai pas en "point-élève» je peux pas [faisant référence à l'histoire familiale de l'élève, évoquée précédemment au cours de l'entretien], on peut pas tout dire même au "point-élève " parce que ça intéresse pas tout le monde et puis toi tu es assistante sociale donc tu vois aussi en dehors du collège tu vois la famille, en tant qu'assistante sociale tu peux pas perdre le contact avec la famille 
c'est important la confiance que tu as construite avec la famille il faut garder la main sur tout cela sur ce contact privilégié sur cette confiance [motif partagé par le collectif professionnel des assistantes sociales] ici si tu dis tout au " point-élève " [motif suggéré par le collectif du dispositif] tu les trahis en quelque sorte et après tu vas ramer pour les revoir.

Dans la compréhension du DS de l'élève et du décrochage professionnel de l'enseignant, ces concepts (knotworking et de boundary crossing) permettent, selon nous, de mieux saisir le poids des tensions de l'activité dans le processus de co-construction du sens et de l'efficience du travail scolaire et du travail enseignant. Ce processus se révèle complexe, sur la base d'ajustements permanents et imprévisibles, échappant à toute logique causaliste.

\section{Vers une redéfinition du décrochage scolaire des élèves et du décrochage professionnel des enseignants}

Le discours institutionnel considère les jeunes comme décrocheurs dès lors qu'ils ont quitté le système éducatif "sans diplôme ou uniquement avec le brevet et ne sont pas en formation" (Direction de l'Évaluation, de la Prospective et de la Performance). En se centrant sur les résultats scolaires de l'élève, cette définition du DS autorise la comparaison de flux par établissements, par régions, par pays en fonction de diverses variables souvent externes. De la sorte, elle apparaît comme un outil indispensable pour orienter les politiques éducatives. Mais elle se révèle insuffisante pour expliquer le processus qui conduit au DS.

Si les nombreuses études menées sur le sujet, notamment sociologiques et didactiques, permettent de mieux connaitre les élèves présentant des risques de DS, il nous semble pertinent de les compléter par d'autres recherches portant la focale sur l'activité in situ de ces élèves pour une compréhension fine du phénomène. L'entrée " orientée activité " proposée dans cet article cherche à atteindre ce but en considérant l'activité de l'élève comme singulière, en interdépendance avec celle de l'enseignant, pouvant faire lui-même l'objet d'un risque de décrochage professionnel.

Dans cette perspective, les conclusions provisoires des travaux font percevoir le DS au travers d'un délitement du processus de co-construction de l'efficience et du sens du travail scolaire, conjointement par l'enseignant et l'élève.

Ce processus est marqué par cinq caractéristiques fortes. Il est a) social, les expériences scolaires, pour être significatives pour l'élève, nécessitant de s'ancrer dans une dimension socio-culturelle reconnue pour lui permettre de s'engager dans des interactions sensées avec l'enseignant; b) inscrit dans une activité de mise en lien entre les expériences passées, présentes et à venir, l'école ne permettant sans doute pas assez à l'élève de construire des passerelles entre les différents moments de sa scolarité, voire avec ses expériences extrascolaires; c) lié au gain d'efficience, la persistance de l'élève dans le travail scolaire étant inséparable de progrès; d) impliquant une maîtrise du collectif d'élèves pour que les règles implicites de ce collectif ne prévalent pas sur celles suggérées par l'enseignant; e) impliquant une revitalisation des collectifs d'enseignants dans des systèmes d'organisation évolutifs, avec l'objectif volontariste de « reprise en main de leur 
métier " par les enseignants eux-mêmes, comme une « riposte » au risque de décrochage professionnel (Clot, 2008).

Dans la lutte contre le DS, la complexité tient essentiellement à la simultanéité de ces caractéristiques, impliquant dès lors un traitement global, mais également individualisé du phénomène au regard de la singularité des situations rencontrées. Ainsi, le processus de DS ne peut plus être appréhendé uniquement comme un processus identifiable à partir de tendances ou de caractéristiques générales et communes, mais au contraire dans ce qu'il a de particulier en référence à l'idiosyncrasie des situations et au sens qu'elles revêtent pour les acteurs.

\section{BIBLIOGRAPHIE}

Assude, T., Feuilladieu, S., \& Dunand, C. (2015). Conditions d'évolution du rapport au savoir mathématique de jeunes « décrocheurs ». Carrefours de l'éducation, 40, 167-182. DOI 10.3917/ cdle.040.0167

Baker, J.A., Derrer, R.D., Davis, S.M., Dinklage-Travis, H.E., Linder, D.S., \& Nicholson, M.D. (2001). The flip side of the coin: Understanding the schools's contribution to dropout and completion. School Psychology Quarterly, 16(4), 406-426.

Bautier, E., \& Rochex, J.Y. (1997). Apprendre : des malentendus qui font la différence. In J.P. Terrail (Ed.), La scolarisation de la France. Critique de l'état des lieux (pp. 105-122). Paris : la Dispute.

Bautier, E. (2003). Décrochage scolaire. Genèse et logique des parcours. VEI-Enjeux, 132, 30-45.

Blaya, C. (2013). Le décrochage scolaire dans les pays de l'OCDE. Regards croisés sur l'économie, 2, 69-80.

Blaya, C. (2014). Expérimentation d'un programme d'intervention en collège (Trait d'union). Accrochage des élèves en formation des enseignants. Communication orale au colloque international "Prévention du décrochage scolaire et formation professionnelle », Draguignan, 12 et 13 juin 2014.

Blaya, C., \& Hayden, C. (2003). Constructions sociales et décrochages scolaires et absentéismes en France et en Angleterre. LARSEF/Observatoire européen de la violence scolaire.

Bonnery, S. (2007). Comprendre l'échec scolaire. Élèves en difficultés et dispositifs scolaires. Paris, France : La Dispute.

Broccolochi, S. (2000). Désagrégation des liens pédagogiques et situations de rupture. VEI-Enjeux, $122,36-47$.

Brown, T.M., \& Rodriguez, L.F. (2009). School and the Co-Construction of Dropout. International Journal of Qualitative Studies in Éducation, 22(2), 221-242.

Bruno, F., Méard, J., \& Walter, E. (2014). Les dispositifs français de lutte contre le décrochage scolaire en collège : ce qui est prescrit et ce qui est mis en œuvre. Orientation scolaire et professionnelle, 42/4. http://osp.revues.org/4183. DOI : 10.4000/osp.4183 
Cairns, R.B., Cairns, B.D., \& Neckerman, H.J. (1989). Early school dropout: Configurations and determinants. Child Development, 60, 1437-1452.

Clot, Y. (1999). La fonction psychologique du travail. Paris : Presses Universitaires de France.

Clot, Y. (2008). Travail et pouvoir d'agir. Paris, France : Presses Universitaires de France.

Davezies, P. (2009). Pouvoir d'agir, travail et santé mentale. Actes du Congrès de l'Association Française de Sociologie, Paris, France.

Durand, M., de Saint-Georges, I., \& Meuwly-Bonte, M. (2006). Le curriculum en formation des adultes : argumentation pour une approche « orientée-activité ». In F. Audigier, M. Crahay, \& J. Dolz (Eds.), Curriculum, Enseignement et pilotage (pp. 185-202). Louvain-la-Neuve: De Boeck Supérieur.

Engeström, Y. (2001). Expansive learning at work: toward an activity theoretical reconceptualization. Journal of Education and Work, 14, 133-156.

Engeström, Y. (2004). New forms of learning in co-configuration work. Journal of Workplace Learning, 16, 11-21.

Entwisle, D.R. (1990). Shoools and the adolescent. In S.S. Feldman \& G.R. ELLIOTT (Eds.), At the threshold: The developping adolescent (pp. 197-224). Cambridge, MA : Harvard University Press.

Flavier, E. (2014). Une expérience d'entreprise virtuelle au collège. In E. Flavier \& S. Moussay (Eds.). Répondre au décrochage scolaire. Expériences de terrain (pp. 127-138). Bruxelles, Belgique : De Boeck.

Flavier, E., \& Moussay, S. (2014). Répondre au décrochage scolaire. Expériences de terrain. Bruxelles, Belgique : De Boeck.

Flavier, E., Moussay, S., \& Méard, J. (2015). Les initiatives individuelles au cœur des dispositifs collectifs locaux de prévention du décrochage scolaire. Les dossiers des sciences de l'éducation, 33, 175-192.

Gilles, J. L., \& Tièche Christinat, C. (2013). Alliances éducatives et accrochage scolaire. Éducation \& Formation, 9-14.

Giust-Despraires, F. (1996). L'identité comme processus, entre liaison et déliaison. Éducation permanente, 128, 63-70.

Houlfort, N., \& Sauve, F. (2010). Santé psychologique des enseignants de la Fédération autonome de l'enseignement. Montréal, Québec : École nationale d'administration publique.

Hugon, M.A. (2010). Lutter contre le décrochage scolaire : quelques pistes pédagogiques ». Informations sociales, 161, 36-45.

Janosz, M. (2000). L'abandon scolaire chez les adolescents : perspective Nord-Américaine. VEI enjeux, 122, 105-127.

Kahn, S. (2012). Et si les malentendus sociocognitifs faisaient ou défaisaient l'effet-maître? Questions Vives, 6(18), 57-72. DOI : 10.4000/questionsvives.1150

Karsenti, T., \& Collin, S. (2009). L'autre décrochage scolaire. Formation et Profession, 16(1), 2-6.

Lee, V.E., \& Burkam, D.T. (2003). Dropping out of high school: The role of school organization and structure. American Educational Research Journal, 40(2), 353-393.

Leontiev, A.N. (1984). Activité, conscience, personnalité. Paris, France : Éditions du Progrès. 
Margolinas, C., \& Laparra, M. (2009). Analyse de situations et production des inégalités scolaires. Premier colloque de didactique comparée : Où va la didactique comparée ? Genève, janvier 2009.

Margolinas, C., \& Laparra, M. (2011). Les savoirs transparents dans le travail des professeurs à l'école primaire. In J.-Y. Rochex, \& J. Crinon, (Eds.). La construction des inégalités scolaires (pp. 19-32). Rennes : Presses Universitaires de Rennes.

Marlot, C., \& Toullec-Théry, M. (2011). Caractérisation didactique des gestes de l'aide ordinaire à l'école élémentaire : une étude comparative de deux cas didactiques limite en mathématiques. Éducation \& didactique, 5(3), 7-32.

Méard, J. (2014). La co-construction de sens dans les interactions entre l'enseignant et les élèves à risque de décrochage. In E. Flavier \& S. Moussay (Eds.). Répondre au décrochage scolaire. Expériences de terrain (pp. 39-50). Bruxelles, Belgique : De Boeck.

Millet, M., \& Thin, D. (2005). Ruptures scolaires. L'école à l'épreuve de la question sociale. Paris: Presses Universitaires de France.

Moussay, S., \& Flavier, E. (2014). L'entretien d'autoconfrontation : la prise en compte du point de vue de l'élève pour développer l'activité en classe. Revue canadienne de l'éducation, 37(1), 96-119.

Mukamurera, J., \& Bouthiette, M. (2008). Rester dans l'enseignement ou quitter ? Portrait de la situation et motivation des enseignants. Séminaire « Pourquoi et comment soutenir l'insertion professionnelle de nouveaux enseignants au Québec ? Résultats de recherche et pistes d'action. " Québec : Université de Sherbrooke.

Poyet, F. (2009). Impact des TIC dans l'enseignement : une alternative pour l'individualisation? Dossier d'actualité, 41. Lyon : Institut Français d'Éducation.

Roger, D. (2007). Refaire son métier. Essais de clinique de l'activité. Toulouse : Érès.

Sanchez, E. (2009). Jeux sérieux : Peut-on apprendre les maths en détruisant des zombies? MathémaTICE, 15. En ligne (consulté le $1^{\mathrm{er}}$ mai 2016) : http://revue.sesamath.net/spip.php? article213.

Schaumburg, H., Prasse, D., Tschackert, K., \& Blömeke, S. (2007). Lernen in Notebook- Klassen. Enbericht zur Evaluation des Projektes “1000 mal 1000 : Notebooks im Schulranzen”. Schulen ans Netz.

Thibert, R., (2013). Le décrochage scolaire : diversité des approches, diversité des dispositifs. Dossier d'actualité Veille et Analyses IFE $n^{\circ}$ 84. Lyon, France : ENS de Lyon.

Toullec-Théry, M., \& Marlot, C. (2013). Les déterminations du phénomène de différenciation didactique passive dans les pratiques d'aide ordinaire à l'école élémentaire. Revue française de pédagogie, 182, 41-54.

Vygotski, L.S. (1934/1997). Pensée et langage (3e édition). Paris, France : La Dispute.

Yvon, F., \& Durand, M. (Eds.) (2012). Réconcilier recherche et formation par l'analyse de l'activité. Bruxelles : De Boeck.

\section{RÉSUMÉS}

Actuellement, les politiques éducatives portent une attention accrue à la prévention et la lutte contre le décrochage scolaire, notamment parce qu'il représente un indicateur de l'état de santé des systèmes éducatifs. Les nombreuses études sur le sujet, principalement sociologiques, permettent de comprendre le phénomène comme un processus complexe et multifactoriel de 
désadhésion au travail scolaire. Dans leur prolongement, cet article, sur fond d'études empiriques, se donne pour ambition d'appréhender la problématique de la lutte contre le décrochage scolaire à partir d'une approche dite "orientée activité ", c'est-à-dire au plus près des acteurs, de leurs pratiques et des significations qu'ils leur accordent en contexte de travail. Nous déclinerons trois hypothèses spécifiques de ce courant: l'hypothèse d'un développement par le sens et l'efficience, l'hypothèse d'un développement par des motifs concurrents, l'hypothèse d'un développement dans un "système d'activité ". L'adoption de ces hypothèses offre de nouvelles perspectives d'étude du phénomène dans une visée développementale, soutenant ainsi l'accroissement du pouvoir d'agir des professionnels de l'éducation au profit d'un meilleur accrochage scolaire des élèves et professionnel des enseignants.

Education policies are currently paying increasing attention to the prevention of and the fight against pupils dropping out of school. This is notably because the school dropout rate is considered to be an indicator of an education system's state of health. Numerous (essentially sociological) studies on this subject allow us to understand the phenomenon as a complex and multifactorial process of non-adherence to school work. Following on from these studies, this article, supported by empirical evidence, seeks to better understand the issues associated with a reduction in the school dropout rate from a "directed activity" approach that is as close as possible to the actors, their practices and the significance they accord them within the work context. We will develop three specific hypotheses from this approach: the hypothesis of development through sense and efficiency, the hypothesis of development through concurrent motives and the hypothesis of development in an "activity system". The adoption of these hypotheses offers new perspectives to study the school dropout phenomenon in a developmental direction, thus supporting the increased empowerment of education professionals in favour of increased school attendance for pupils and professional satisfaction for teachers.

\section{INDEX}

Keywords : school dropout, cultural-historical activity theory (CHAT), sense co-construction, efficiency, collective work, education

Mots-clés : décrochage scolaire, théorie historico culturaliste de l'activité (CHAT), coconstruction du sens, efficience, travail collectif, enseignement

\section{AUTEURS}

\section{ÉRIC FLAVIER}

LISEC EA 2310, Université de Strasbourg, Strasbourg, France. ESPE de l'académie de Strasbourg, 141 avenue de Colmar, BP 40102, 67024 STRASBOURG Cedex eric.flavier@unistra.fr

\section{JACQUES MÉARD}

LASALé, HEP du canton de Vaud, Lausanne, Suisse HEP Vaud, Avenue de Cour 33, 1007 Lausanne, Suisse jacques.meard@hepl.ch 